\title{
A Phase II Study of Avelumab Monotherapy in Patients with Mismatch Repair-Deficient/Microsatellite Instability-High or POLE-Mutated Metastatic or Unresectable Colorectal Cancer
}

\author{
Jwa Hoon Kim, MD ${ }^{1}$ \\ Sun Young Kim, MD, PhD' \\ Ji Yeon Baek, MD, PhD² \\ Yong Jun Cha, MD, $\mathrm{PhD}{ }^{2}$ \\ Joong Bae Ahn, MD, $\mathrm{PhD}^{3}$ \\ Han Sang Kim, MD, PhD ${ }^{3}$ \\ Keun-Wook Lee, MD, PhD ${ }^{4}$ \\ Ji-Won Kim, MD, PhD ${ }^{4}$ \\ Tae-You Kim, MD, PhD ${ }^{5}$ \\ Won Jin Chang, MD, $P h D^{6}$ \\ Joon Oh Park, MD, $\mathrm{PhD}{ }^{7}$ \\ Jihun Kim, MD, PhD \\ Jeong Eun Kim, MD, PhD ${ }^{1}$ \\ Yong Sang Hong, MD, $\mathrm{PhD}^{1}$ \\ Yeul Hong Kim, MD, $\mathrm{PhD}^{6}$ \\ Tae Won Kim, MD, PhD'
}

*A list of author's affiliations appears at the end of the paper.

\begin{abstract}
Purpose
We evaluated the efficacy and safety of avelumab, an anti-PD-L1 antibody, in patients with metastatic or unresectable colorectal cancer $(\mathrm{mCRC})$ with mismatch repair deficiency (dMMR)/microsatellite instability-high (MSI-H) or POLE mutations.
\end{abstract}

\section{Materials and Methods}

In this prospective, open-label, multicenter phase II study, 33 patients with $\mathrm{mCRC}$ harboring $\mathrm{dMMR} / \mathrm{MSI}-\mathrm{H}$ or POLE mutations after failure of $\geq 1$ st-line chemotherapy received avelumab $10 \mathrm{mg} / \mathrm{kg}$ every 2 weeks. dMMR/MSI-H was confirmed with immunohistochemical staining (IHC) by loss of expression of MMR proteins or polymerase chain reaction (PCR) for micro-satellite sequences. POLE mutation was confirmed by next-generation sequencing (NGS). The primary endpoint was the objective response rate (ORR) by Response Evaluation Criteria in Solid Tumors ver. 1.1.

\section{Results}

The median age was 60 years, and $78.8 \%$ were male. Thirty patients were dMMR/MSI$\mathrm{H}$ and three had POLE mutations. The ORR was $24.2 \%$, and all of the responders were $\mathrm{dMMR} / \mathrm{MSI}-\mathrm{H}$. For 21 patients with MSI-H by PCR or NGS, the ORR was $28.6 \%$. At a median follow-up duration of 16.3 months, median progression-free survival and overall survival were 3.9 and 13.2 months in all patients, and 8.1 months and not reached, respectively, in patients with MSI-H by PCR or NGS. Dose interruption and discontinuation due to treatment-related adverse events occurred in four and two patients, respectively, with no treatment-related deaths.

\section{Conclusion}

Avelumab displayed antitumor activity with manageable toxicity in patients with previously treated mCRC harboring dMMR/MSI-H. Diagnosis of dMMR/MSI-H with PCR or NGS could be complementary to IHC to select patients who would benefit from immunotherapy.

\section{Key words}

Colorectal neoplasms, Mismatch repair deficiency, Microsatellite instability, POLE mutation, Avelumab

\section{Introduction}

Colorectal cancer (CRC) is one of the leading causes of cancer-related death worldwide and the third most common cancer in Korea [1]. Standard palliative treatment for metastatic or unresectable CRC (mCRC) is fluorouracil-based combination chemotherapy (with oxaliplatin or irinotecan), with or without agents targeting angiogenesis (bevacizumab) or epidermal growth factor receptor (cetuximab). The available therapeutic options for later-line chemotherapy are limited; regorafenib and TAS-102 showed only a modest clinical benefit in these patients. The objective response rate (ORR) with regorafenib and TAS-102 was approximately $1 \%$, and median progression-free survival (PFS) was around 2 months for both treatments $[2,3]$. The long-term outcomes of mCRC are still poor [1], and novel therapeutic approaches are needed.

Growing evidence suggests that patients with $\mathrm{mCRC}$ harboring deficient mismatch repair protein $(\mathrm{dMMR}) /$ microsatellite instability-high (MSI-H) can obtain clinical benefit from immune checkpoint inhibitors (ICIs) [4-7]. Pembroli- 
zumab and nivolumab, which are anti-programmed death 1 (anti-PD-1) inhibitors, improved ORR and PFS in selected patients with dMMR/MSI-H mCRC [4-7]. Failure to repair DNA replication-associated errors in dMMR/MSI-H mCRC is associated with high mutation loads, tumor neoantigen loads, and dense immune cell infiltration [8]. In fact, the whole-exome sequences revealed higher somatic mutation loads (1,782 mutations per tumor) in patients with dMMR/ MSI-H than in patients with proficient MMR (73 mutations per tumor) ( $\mathrm{p}=0.007)$, and a greater density of $\mathrm{CD} 8$-positive lymphocytes and a higher expression of PD-ligand 1 (PD-L1) were observed in patients with $\mathrm{dMMR} / \mathrm{MSI}-\mathrm{H}$ than in patients with proficient MMR [4]. However, the clinical benefit of ICIs is confined to a small proportion of patients, because dMMR/MSI-H is identified in only about 5\% in patients with mCRC [9]. This raises the need to expand the number of potential candidates for immunotherapy.

The POLE gene is located in 12q24.33 and encodes the proofreading (exonuclease) subunit of polymerase epsilon (POLE) with 2,286 amino acids [10]. This POLE mutation has been reported in approximately $3 \%$ of proficient MMR CRC and represents high somatic mutation loads [10]. According to the Cancer Genome Atlas, up to one-quarter of hypermutated CRC carry somatic mutations in POLE [10]. Because high mutation loads are considered a mechanism of the response of dMMR/MSI-H to ICIs, POLE-mutated cancer may also be susceptible to ICIs. However, to date, clinical data regarding the response to ICIs in POLE-mutated cancer are lacking.

Treatment with avelumab, an anti-PD-L1 inhibitor, achieved an ORR of $33 \%$ in patients with previously treated metastatic Merkel cell carcinoma and was approved for the treatment of metastatic Merkel cell carcinoma in early 2017 [11]. Subsequently, avelumab has shown promising antitumor activity in various solid tumors, such as genitourinary tract [12,13], gynecologic [14], and lung [15], but its activity in mCRC harboring dMMR/MSI-H has not been investigated. This study aimed to evaluate the efficacy and safety of avelumab in patients with previously treated CRC with deficient MMR/MSI-H as well as those with POLE mutations.

\section{Materials and Methods}

\section{Study design and patients}

This study is a prospective, open-label, multicenter phase II study conducted as a substudy of the K-MASTER project, a nationwide, government-funded precision medicine initiative [16]. Eligible patients were aged $\geq 20$ years and had histologically or cytologically confirmed metastatic or unresectable adenocarcinoma of the colon or rectum after failure of first-line or later chemotherapy, including fluoropyrimidine, oxaliplatin, or irinotecan, with or without targeted agents (bevacizumab or cetuximab). Patients were enrolled if
dMMR/ MSI-H was confirmed by either immunohistochemistry (IHC) or polymerase chain reaction (PCR) by local test at each site, or if POLE mutation was confirmed by next-generation sequencing (NGS) certified by the Ministry of Food and Drug Safety, Korea. MMR protein was determined to be deficient by loss of expression of one or more of the following on IHC: MLH1, MSH2, MSH6, and PMS2. MSI-H was diagnosed by PCR if two or more microsatellite markers (BAT25, BAT-26, D2S123, D5S346, and D17S250) were detected. POLE mutations included hotspots such as P286R and other sites. Eligible patients had at least one measurable disease, an Eastern Cooperative Oncology Group (ECOG) performance status of 0 or 1 , and adequate organ function. Any prior treatment with anti-PD-1 or PD-L1 inhibitors was not permitted, and prior immunosuppressive treatment or the last dose of chemotherapy should not be administered within 28 days before the first dose of study drug. Prior radiotherapy was permitted if it was not administered to the target lesions selected for this study.

\section{Treatment and evaluation}

Avelumab was administered at $10 \mathrm{mg} / \mathrm{kg}$ intravenously every 2 weeks until disease progression, unacceptable toxicity, or patient refusal occurred. Dose modification was not allowed, but dose delay was permitted at the investigator's discretion in case of clinically significant events. Study treatment was discontinued if there were more than 4 weeks of delay. Response assessment was performed by computed tomography (CT) scan according to Response Evaluation Criteria in Solid Tumors (RECIST) ver. 1.1 every 6 weeks. After the end of treatment, patients were followed up for disease status and survival information every 3 months. The medical histories of all patients were obtained before treatment, including physical examination, complete blood count with differential count, serum chemistry, electrolytes, coagulation, carcinoembryonic antigen, thyroid function test (thyroidstimulating hormone and free thyroxine), urinalysis, electrocardiogram, chest X-ray, CT scan, and other scans if clinically indicated. Adverse events were assessed every cycle according to the National Cancer Institute Common Terminology Criteria for Adverse Events (NCI-CTCAE), version 4.0.

\section{Statistical analysis}

The sample size was calculated using the Simon two-stage optimal design. The target response rate was set to $30 \%$, and a rate of $10 \%$ or below was considered failure, allowing early termination of any ineffective treatment early in the study. With a one-sided type I error of $5 \%$ and a power of 0.8 , the planned study was to proceed in two steps. If a tumor response occurred in at least two patients after the first 10 patients were listed, the study proceeded to the second stage with 19 additional patients. A total of 29 patients were requi-red, and enrollment of 33 patients was planned, 
Table 1. Baseline characteristics of the study patients

\begin{tabular}{|c|c|}
\hline Characteristic & No. $(\%)$ \\
\hline Age, median (range, yr) & $60(25-88)$ \\
\hline \multicolumn{2}{|l|}{ Sex } \\
\hline Male & $26(78.8)$ \\
\hline Female & $7(21.2)$ \\
\hline \multicolumn{2}{|l|}{ ECOG performance status } \\
\hline 0 & $10(30.3)$ \\
\hline 1 & $23(69.7)$ \\
\hline \multicolumn{2}{|l|}{ Primary tumor } \\
\hline Right-sided colon & $22(66.7)$ \\
\hline Left-sided colon & $5(15.2)$ \\
\hline Rectum & $6(18.2)$ \\
\hline \multicolumn{2}{|l|}{ Histology } \\
\hline Well differentiated & $6(18.2)$ \\
\hline Moderately differentiated & $19(57.6)$ \\
\hline Poorly differentiated & $5(15.2)$ \\
\hline Not assessable & $3(9.1)$ \\
\hline \multicolumn{2}{|l|}{$R A S$ status } \\
\hline Wild & $11(33.3)$ \\
\hline Mutant & $20(60.6)$ \\
\hline Not done & $2(6.1)$ \\
\hline \multicolumn{2}{|l|}{$B R A F$ status } \\
\hline Wild & $22(66.7)$ \\
\hline Mutant & $4(12.1)$ \\
\hline Not done & $7(21.2)$ \\
\hline \multicolumn{2}{|l|}{ Sites of metastasis } \\
\hline Liver & $15(45.5)$ \\
\hline Lung & $11(33.3)$ \\
\hline Lymph node, abdomen & $20(60.6)$ \\
\hline Peritoneum/Omentum & $9(27.3)$ \\
\hline Bone & $2(6.1)$ \\
\hline $\begin{array}{l}\text { Family history of cancer in } \\
\text { any first-degree relative }\end{array}$ & $7(21.2)$ \\
\hline \multicolumn{2}{|c|}{ Previous chemotherapy regimen } \\
\hline FOLFOX & $24(72.7)$ \\
\hline FOLFIRI & $15(45.5)$ \\
\hline XELOX & $4(12.1)$ \\
\hline Capecitabine & $10(30.3)$ \\
\hline Others & $2(6.1)$ \\
\hline \multicolumn{2}{|l|}{ Previous targeted treatment } \\
\hline Bevacizuamb & $25(75.8)$ \\
\hline Cetuximab & $2(6.1)$ \\
\hline Prior radiotherapy & $7(21.2)$ \\
\hline \multicolumn{2}{|l|}{ Prior surgery } \\
\hline Primary site resection & $31(93.9)$ \\
\hline Metastasectomy & $9(27.3)$ \\
\hline
\end{tabular}

(Continued)

given a dropout rate of $10 \%$.

The primary endpoint was the ORR by RECIST ver. 1.1; the secondary endpoints included the disease control rate (DCR), PFS, overall survival (OS), and adverse events. DCR
Table 1. Continued

$\begin{array}{lr}\text { Characteristic } & \text { No. }(\%) \\ \text { Prior lines of therapy } & \\ 1 & 16(48.5) \\ 2 & 11(33.3) \\ \geq 3 & 6(18.2)\end{array}$

ECOG, Eastern Cooperative Oncology Group; FOLFOX, 5-fluorouracil, leucovorin, and oxaliplatin; FOLFIRI, 5-fluorouracil, leucovorin, and irinotecan; XELOX, capecitabine and oxaliplatin.

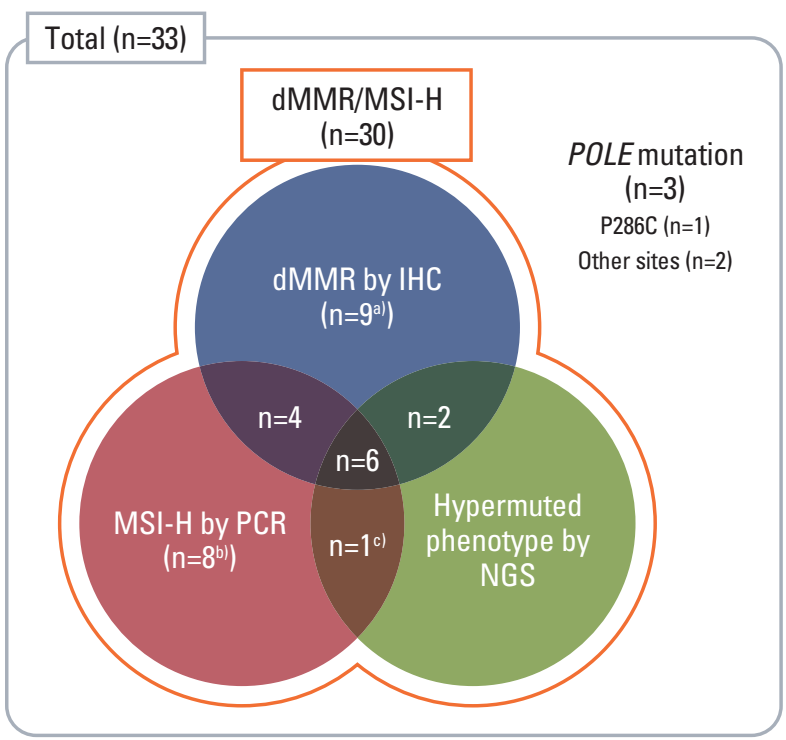

Fig. 1. Status of mismatch repair by immunohistochemistry (IHC) or microsatellite instability (MSI) by polymerase chain reaction $(\mathrm{PCR})$ and POLE mutation. dMMR, mismatch repair deficiency; MSI-H, MSI-high; MSS, microsatellite stable; NGS, next-generation sequencing; $\mathrm{p}-\mathrm{MMR}$, proficient-microsatellite

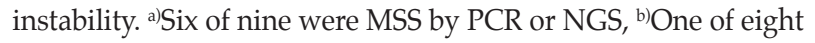
was $\mathrm{p}-\mathrm{MMR}$ by IHC, c) p-MMR by IHC.

was defined as the proportion of patients with complete response (CR), partial response (PR), or stable disease (SD) sustained for $\geq 6$ weeks. PFS was calculated from the first date of avelumab administration to the date of disease progression or death from any cause. OS was calculated from the first date of avelumab administration to the date of death from any cause. Survival rates were estimated using the KaplanMeier method. A two-sided p-value $<0.05$ was considered to indicate statistical significance, and all statistical analyses were performed using IBM SPSS Statistics for Windows ver. 21.0 (IBM Corp., Armonk, NY).

\section{Ethical statement}

All procedures followed were in accordance with the ethi- 


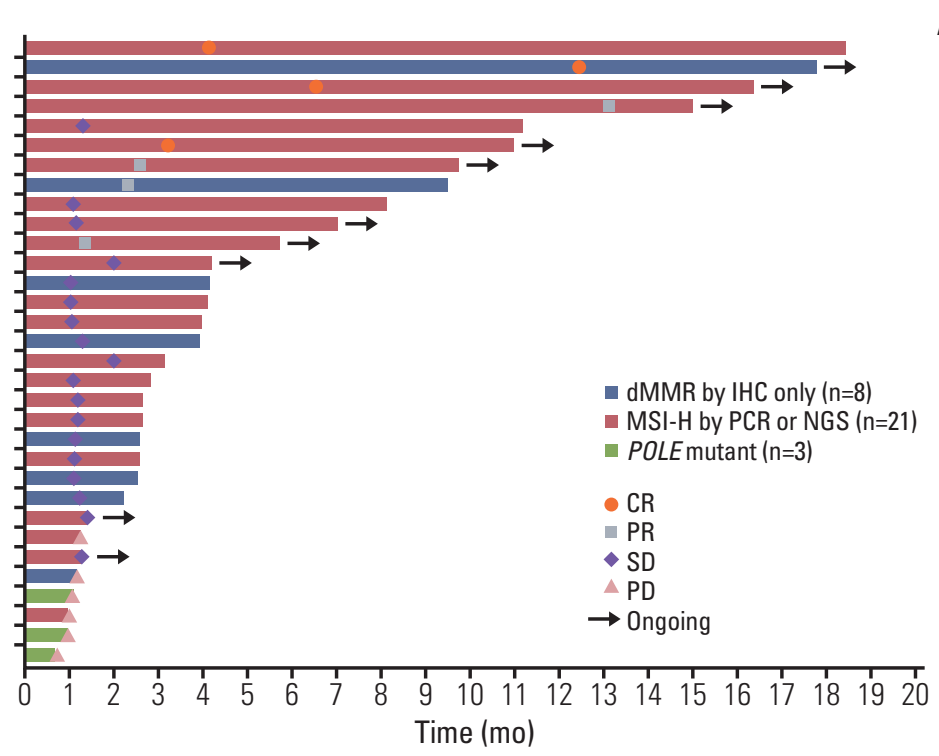

A

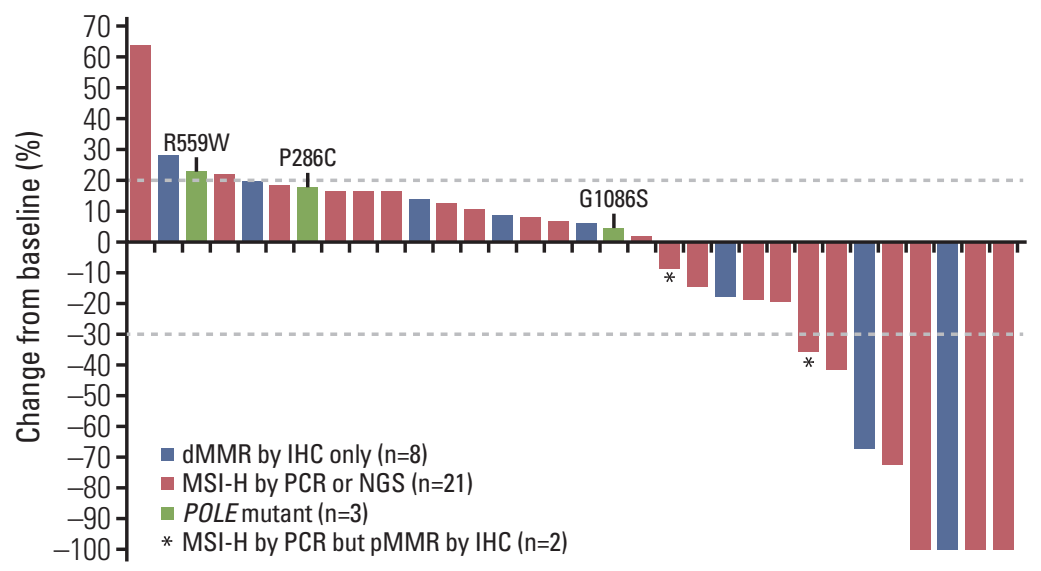

Fig. 2. Antitumor activity of avelumab in patients with metastatic or unresectable colorectal cancer harboring deficient mismatch repair (dMMR)/ microsatellite instability-high (MSI-H) or POLE mutations. (A) Treatment duration of avelumab for all patients. (B) Best change from baseline in target lesion size after avelumab. CR, complete response; IHC, immunohistochemistry; NGS, next-generation sequencing; PCR, polymerase chain reaction; PD, progressive disease; p-MMR, proficient-microsatellite instability; PR, partial response; SD, stable disease.

cal standards of the responsible committee on human experimentation (institutional and national) and the Helsinki Declaration of 1964 and later versions. The study protocol was approved by the institutional review board of each participating center and the Korean Cancer Study Group (KCSG; protocol No. KCSG-CO-17-07). This trial was registered on http:// www.clinicaltrials. gov with the identifier NCT03150706. Informed consent or a substitute was obtained from all patients before inclusion in the study.

\section{Results}

\section{Patient characteristics}

Between May 2017 and April 2019, a total of 34 patients were initially enrolled at seven clinical sites in South Korea; one patient failed the screening tests, and thus, 33 patients with histologically or cytologically confirmed, previously treated, metastatic or unresectable CRC were enrolled in the final analysis. Table 1 summarizes the baseline characteristics of the 33 study patients. The median age was 60 years (range, 25 to 88 ), and $78.8 \%$ were male. Two-thirds of the patients $(\mathrm{n}=22,66.7 \%)$ had right-sided colon cancer, and 20 patients $(60.6 \%)$ had RAS mutation. Seven patients had a family his- 
Table 2. Clinical response to avelumab monotherapy

\begin{tabular}{|c|c|c|c|c|}
\hline \multirow{2}{*}{ Response } & \multicolumn{2}{|c|}{ All patients $(n=33)$} & \multicolumn{2}{|c|}{ MSI high by PCR or NGS $(n=21)$} \\
\hline & No. $(\%)$ & $95 \% \mathrm{CI}(\%)$ & No. $(\%)$ & $95 \%$ CI (\%) \\
\hline Complete response & $4(12.1)$ & $0.97-23.2$ & $3(14.3)$ & $0-29.3$ \\
\hline Partial response & $4(12.1)$ & $0.97-23.2$ & $3(14.3)$ & $0-29.3$ \\
\hline Stable disease & $18(54.5)$ & $37.5-71.5$ & $13(61.9)$ & $41.1-82.7$ \\
\hline Progressive disease & $6(18.2)$ & $5.0-31.4$ & $2(9.5)$ & $0-22.0$ \\
\hline Not assessable $e^{a)}$ & $1(3.0)$ & $0-8.8$ & 0 & - \\
\hline Objective response rate & $8(24.2)$ & $9.4-38.6$ & $6(28.6)$ & $9.3-47.9$ \\
\hline Disease control rate & $26(78.8)$ & $64.9-92.7$ & $19(90.5)$ & 77.9-103 \\
\hline
\end{tabular}

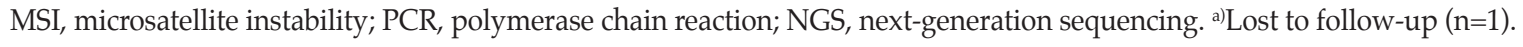
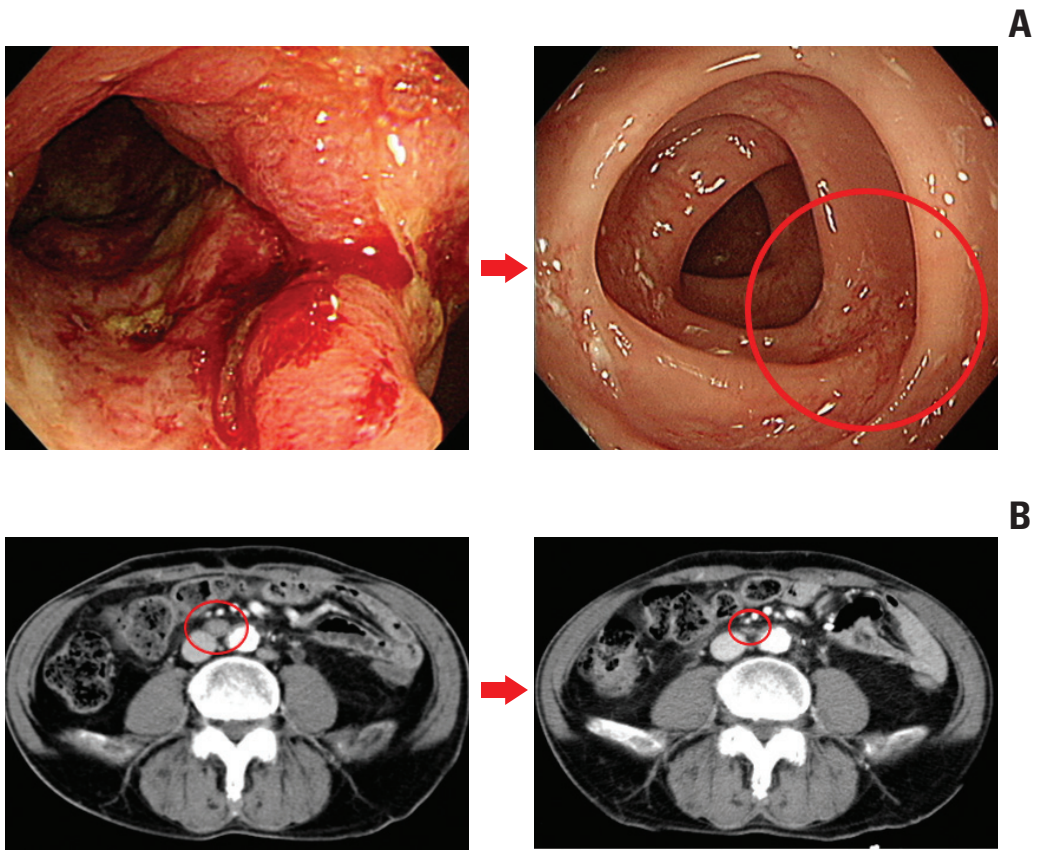

Fig. 3. Representative images of two patients who achieved partial response during avelumab monotherapy. (A) Endoscopic images of a 44-year-old man with initially metastatic T-colon cancer harboring microsatellite instability-high (MSI-H) by both immunohistochemistry (IHC) and next-generation sequencing. (B) Computed tomography scans of 75-year-old man with recurrent rectal cancer harboring MSI-H by IHC.

tory of cancer in a first-degree relative.

\section{Deficient MMR or MSI high and POLE mutation}

Among the 33 patients, 30 patients had dMMR/MSI-H CRC, and three patients had POLE-mutated CRC. POLE mutation was identified at the P286C, R559W, and G1086S sites. NGS revealed hypermutated phenotype CRC in only one patient with a P286C mutation, but the other sites (R559W and G1086S) were not associated with high tumor mutation burden (TMB) in each different NGS panel (S1 Table).

Among 30 patients with dMMR/MSI-H, IHC, and PCR were performed both in 23 patients, respectively. Both tests at the same time were performed in 16 patients, among whom
$10(62.5 \%)$ showed concordance between IHC and PCR. NGS, which was not mandatory for detecting dMMR/ MSI$\mathrm{H}$, was performed in 12 patients with dMMR/MSI-H.

The distribution of patients according to different detection methods is shown in Fig. 1. Twenty-one patients were diagnosed with dMMR by IHC, and 19 were diagnosed with MSI-H by PCR. Discordance between the IHC and PCR results was found as follows: six of nine patients with dMMR by IHC only showed microsatellite stable (MSS) CRC by PCR or NGS, and one of eight patients with MSI-H by PCR only showed proficient MMR by IHC. One patient who was diagnosed as MSI-H by PCR and NGS was diagnosed as proficient MMR by IHC. 
A

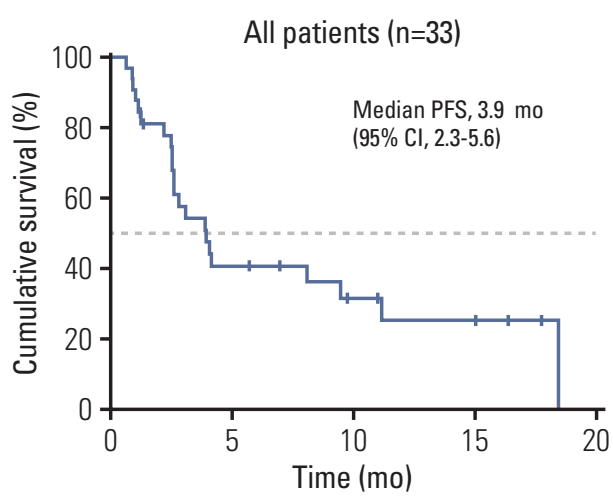

C

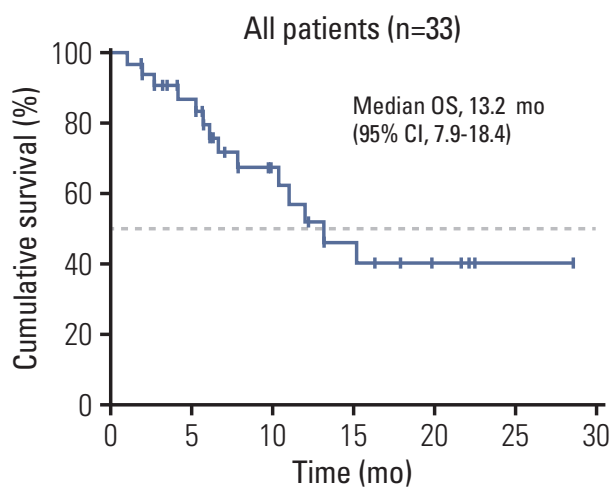

Patients with MSI-H by

B
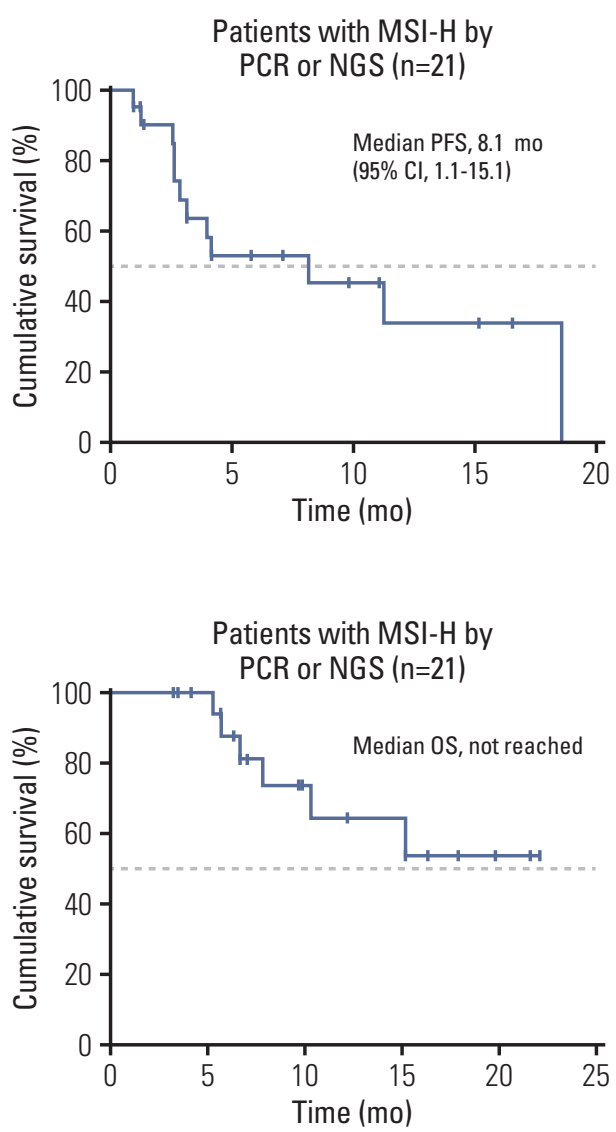

Fig. 4. Median progression-free survival (PFS) (A, B) and overall survival (OS) (C, D) in all patients (A, C) and patients with microsatellite instability high (MSI-H) (B, D) by polymerase chain reaction (PCR) or next-generation sequencing (NGS). CI, confidence interval.

\section{Clinical response to avelumab}

Avelumab was discontinued mainly due to disease progression $(n=22,66.7 \%)$, followed by loss to follow-up ( $n=1$, $0.03 \%$ ); the remaining 10 patients were treated with ongoing avelumab (Fig. 2A). The median time to response was 4.2 months, and the median duration of response was 13.9 months (Fig. 2A). Among the 33 patients, four (12.1\%) had CR, four (12.1\%) had PR, 18 (54.5\%) had SD, $6(18.2 \%)$ had progressive disease (PD), and one (3.0\%) was not assessable (Table 2). The ORR and DCR were $24.2 \%$ and $78.8 \%$, respectively. Of 21 patients with MSI-H by PCR or NGS, three (14.3\%) had CR, three (14.3\%) had PR, 13 (61.9\%) had SD, and two $(9.5 \%)$ had PD (Table 2). The ORR and DCR were $28.6 \%$ and $90.5 \%$, respectively. All responders had dMMR/ MSI-H CRC, and no patients with a POLE mutation achieved response.

The best percentage changes from baseline in target lesion size are shown in Fig. 2B. Six of eight patients with CR or PR showed MSI-H by PCR or NGS. Among six patients with PD, two patients with POLE mutations (G1086S and P286C) had PD based on unequivocal progression of nontarget lesions, and all three patients with POLE mutations had PD without any tumor shrinkage. One patient with proficient MMR by IHC only but MSI-H by PCR achieved PR. Representative images of two responders are shown in Fig. 3.

Given the discrepancies between IHC and PCR results and the need to investigate their association with the response to avelumab, IHC results were separately reviewed in available eight of nine patients with dMMR by IHC only at each clinical site. dMMR was revised to proficient MMR by IHC in five patients, who also showed MSS by PCR or NGS. Four of them did not achieved response; however, the other patient achieved CR despite proficient MMR revised after review of the IHC result and MSS by PCR.

\section{PFS and OS}

With a median follow-up duration of 16.3 months (95\% confidence interval [CI], 5.6 to 27.1 months), median PFS was 3.9 months (95\% CI, 2.3 to 5.6 months) in all patients and 8.1 months (95\% CI, 1.1 to 15.1 months) in patients with MSI-H by PCR or NGS (Fig. 4A and B). Median OS was 13.2 months (95\% CI, 7.9 to 18.4 months) in all patients and not reached in patients with MSI-H by PCR or NGS (Fig. 4C and D). Overall, the 12-month PFS and OS rates were $36.4 \%$ and $66.7 \%$, 
Table 3. Treatment-related adverse events

\begin{tabular}{lcc} 
& \multicolumn{2}{c}{ All patients (n=33, 100\%) } \\
\cline { 2 - 3 } Event & Any grade & Grade $\geq 3$ \\
Any TRAE & $24(72.7)$ & $6(18.2)$ \\
Myalgia & $6(18.2)$ & 0 \\
Chills & $5(15.2)$ & 0 \\
\hline Infusion-related reaction & $5(15.2)$ & 0 \\
Pruritus & $5(15.2)$ & 0 \\
Thyroid dysfunction & $4(12.1)$ & 0 \\
Skin rash & $4(12.1)$ & 0 \\
\hline Diarrhea & $3(9.1)$ & $2(6.1)$ \\
Fever & $3(9.1)$ & 0 \\
Increased AST or ALT & $3(9.1)$ & 0 \\
Hypomagnesemia & $3(9.1)$ & 0 \\
Fatigue & $2(6.1)$ & 0 \\
Anorexia & $2(6.1)$ & 0 \\
\hline Hyperglycemia & $2(6.1)$ & $1(3.0)$ \\
Increased amylase or lipase & $1(3.0)$ & $2(6.1)$ \\
\hline Nausea & $1(3.0)$ & 0 \\
\hline Sweating & $1(3.0)$ & 0 \\
\hline Dry skin & $1(3.0)$ & 0 \\
Anemia & $1(3.0)$ & 0 \\
\hline Hyperbilirubinemia & $0(0.0)$ & $1(3.0)$ \\
\hline Values are presented as number $(\%)$. TRAE, treatment-related \\
adverse event; AST, aspartate aminotransferase; & ALT, alanine \\
aminotransferase. & & \\
\hline
\end{tabular}

respectively, and $47.6 \%$ and $76.2 \%$, respectively, in patients with MSI-H by PCR or NGS.

\section{Treatment-related adverse events}

Treatment-related adverse events (TRAEs) with avelumab are shown in Table 3. TRAEs of any grade were observed in 24 patients $(72.7 \%)$. Common TRAEs of any grade included myalgia $(n=6,18.2 \%)$, chills $(n=5,15.2 \%)$, infusion-related reaction $(n=5,15.2 \%)$, pruritus $(n=5,15.2 \%)$, thyroid dysfunction $(n=4,12.1 \%)$, and skin rash $(n=4,12.1 \%)$. Grade 3 or 4 TRAEs occurred in six patients (18.2\%). Dose interruption due to TRAEs occurred in four patients $(12.1 \%)$ : grade 3 hyperglycemia $(n=1)$, grade 3 lipase increase $(n=1)$, grade 2 aspartate aminotransferase increase $(n=1)$, and grade 2 fever $(n=1)$. There were two discontinuations of treatment due to treatment-related grade 3 hyperbilirubinemia $(n=1)$ and probable treatment-related grade 4 tumor bleeding $(n=1)$ after response to avelumab as a serious adverse event. There were no deaths due to TRAE.

\section{Discussion}

In this open-label, multicenter, phase II study, avelumab showed promising antitumor activity and manageable tox- icity in patients with $\mathrm{mCRC}$ harboring $\mathrm{dMMR} / \mathrm{MSI}-\mathrm{H}$ or POLE mutation. The ORR and median PFS were $24.2 \%$ and 3.9 months, respectively, and six of eight responders were continuing avelumab treatment with durable response at the end of the analysis. Of note, the ORR and median PFS were $28.6 \%$ and 8.1 months, respectively, in patients with MSI$\mathrm{H}$ by PCR or NGS, which were thought to be more reliable methods of determining the MSI status than IHC. Although no patients with POLE mutations had response to avelumab, the limitations of small sample size and variation in mutation sites need to be taken into account. TRAEs of any grade and TRAEs of grade 3 or 4 were observed in $72.7 \%$ and $18.2 \%$ of patients, respectively, which was consistent with previous studies [17]. There were two discontinuations of treatment, one because of a TRAE and the other because of a serious adverse event, probably related to treatment. There were no treatment-related deaths.

The efficacy of avelumab (an anti-PD-L1 inhibitor) for mCRC with dMMR/MSI-H, specifically in patients with MSI-H by PCR or NGS, is comparable to that of pembrolizumab and nivolumab (anti-PD-1 inhibitors) in this setting. In the KEYNOTE-016 trial, only patients with MSI-H CRC had the objective response to pembrolizumab (ORR 40\%), whereas none of those with MSS CRC had the objective response [4]. A subsequent multicenter trial of pembrolizumab for dMMR/MSI-H CRC, the KEYNOTE-164 phase II study, resulted in an ORR of 33\% and a median PFS of 4.1 months; the 12-month PFS and OS rates were 34\% to $41 \%$ and $72 \%$ to $76 \%$, respectively, according to prior line of treatment [6,7]. Nivolumab also showed promising antitumor activity in terms of ORR (31.1\%) in dMMR/MSI-H CRC, and the 12-month PFS and OS rates were 50\% and 73\%, respectively [5]. All these favorable results in $\mathrm{dMMR} / \mathrm{MSI}-\mathrm{H}$ CRC contrast sharply with those of later-line conventional chemotherapy for overall mCRC with treatments such as regorafenib or TAS-102, which resulted in ORR of only $1 \%$ and median PFS of around 2 months [2,3].

Several phase I results from the JAVELIN Solid Tumor Trials have shown promising ORRs and disease stabilization with avelumab in various types of advanced tumors. Specifically, among 53 patients with metastatic or locally advanced previously treated solid tumors, four (8\%) achieved responses and $30(57 \%)$ had SD [18]. The ORR with avelumab ranged from $6.7 \%$ to $18.2 \%$, depending on tumor type such as metastatic or unresectable previously treated renal cell carcinoma [12], urothelial carcinoma [13], non-small cell lung cancer [15], and ovarian cancer [14]. In a phase II study of 88 patients with chemotherapy-refractory metastatic Merkel cell carcinoma [11], the ORR with avelumab was 33\%, including a CR rate of $11.4 \%$. To date, avelumab has been approved for treatment of previously treated metastatic Merkel cell carcinoma, urothelial carcinoma, and renal cell carcinoma in combination with axitinib. Several explorations to expand 
its use in various clinical settings are ongoing. The present study adds to the evidence of clinical activity of avelumab by showing durable objective responses in $\mathrm{mCRC}$ with $\mathrm{dMMR}$ / MSI-H.

$\mathrm{dMMR} / \mathrm{MSI}-\mathrm{H}$ is an established biomarker for the efficacy of ICIs in $\mathrm{mCRC}$, and its predictive value has also been confirmed in other various tumors. MSI-H metastatic gastric cancer (mGC) had a higher ORR with pembrolizumab than did MSS mGC (57\% vs. 9\%) in the KEYNOTE-059 study [19]. Recently, the clinical benefit of pembrolizumab was demonstrated among patients with dMMR/MSI-H non-CRC, including endometrial cancer, gastric cancer, cholangiocarcinoma, and pancreatic cancer in terms of ORR $(34.3 \%)$ and median PFS (4.1 months) [20]. Although the exact mechanism is unknown, there are several proposed explanations for T-cell checkpoint blockade [21]. Since dMMR/MSI-H results in diverse neoantigens, T-cell epitopes that are newly formed as a consequence of tumor-specific mutations, which can increase neoantigen-driven T-cell response. Another possible explanation is that $\mathrm{dMMR} / \mathrm{MSI}-\mathrm{H}$ is associated with the activation of signaling pathways through altered cytokines or chemokines, resulting in the tumor microenvironment becoming more inflamed. Cellular stress induced by dMMR/ MSI-H promotes innate immune cells, such as T cells and natural killer cells, or tumor recognition.

However, not all patients with mCRC harboring dMMR/ MSI-H respond to ICIs, and the TMB varies even within $\mathrm{dMMR} / \mathrm{MSI}-\mathrm{H}$ mCRC. The updated analysis from the initial single-center study showed an ORR of 50\% [4], but subsequent multicenter phase II trials revealed ORR of around $30 \%$ [5-7]. In the present study, the ORR was $28.6 \%$ in patients with MSI-H by PCR or NGS. Approximately 37 to 41 mutations per megabase may be a cutoff value, and low TMB was significantly associated with poor response to ICIs and worse PFS within the dMMR/MSI-H population, which may be an explanation for the heterogeneity in response in recent clinical trials of dMMR/MSI-H CRC [22]. Moreover, substantial genomic variation is observed within dMMR/MSI-H tumors. In particular, the genome-wide intensity of MSI and the accumulation of insertion-deletion mutational loads are responsible for a wide diversity of clinical benefits with ICIs [23], and the activated WNT/ $\beta$-catenin signaling pathway is associated with immune escape, despite a high TMB and high numbers of tumor-infiltrating lymphocytes [24].

In fact, a considerable portion of the primary resistance of mCRC treated with ICIs may be due to misdiagnosis of $\mathrm{dMMR} / \mathrm{MSI}-\mathrm{H}$. One study showed that three of five patients $(60 \%)$ who had PD at the first evaluation were reassessed as MSS by central review with PCR, contrary to the diagnosis of dMMR/MSI-H by local assessment [25]. In the CheckMate-142 study, there were discrepancies between local and central assessments in 14 of 74 patients (19\%) [5]. Most of the patients with assessments $(10 / 14)$ were initially determined as dMMR by IHC at the local laboratory, but central review with PCR reclassified them as MSS [5]. In this study, there were some discrepancies between IHC and PCR results, and six of nine patients with dMMR by IHC only showed MSS by PCR or NGS, leading to different ORRs depending on how dMMR/MSI-H CRC was defined. After review of the available IHC results in eight of these patients, five patients were revised to proficient MMR, while their PCR or NGS showed all MSS. The reliability and reproducibility of IHC results have always been a concern, because IHC results are largely affected by intra- and interobserver variation and tissue preservation status [26], and local assessment without central review contributed to these results. Likewise, in the recent phase II study with a similar design, differences in ORRs with avelumab in patients with previously treated endometrial cancer were observed between dMMR determined by IHC only and MSI-H determined by NGS (26.7\% vs. 30\%) [27]. dMMR/MSI-H could be misdiagnosed if IHC results are not supported by PCR or NGS, so the IHC test alone should be carefully interpreted by experienced pathologists. Further, IHC tests on tumor samples achieved after chemoradiotherapy, old samples, or poorly preserved samples are associated with a high risk of unreliable results.

POLE-mutated CRC has been characterized by young age, male predominance, right-sided $\mathrm{CRC}$, earlier stage of disease, and excellent prognosis [10]. POLE has a crucial role in chromosomal DNA replication by its proofreading capacity and is known to be mutually exclusive with dMMR/MSI$\mathrm{H}$ [10]. Because of high immunogenicity and enrichment of mutation-associated neoantigens, POLE-mutated cancer has been considered a candidate for treatment with ICIs. However, there are limited data, and there is only one case report [28] of a patient with mCRC harboring a POLE mutation (V411L) and MSS. This patient had a response after three cycles of pembrolizumab, and CD8 infiltrating lymphocytes with PD-1 expression were observed in the primary colon tumor. All responders in previous reports had mutations at the P286R or V411L sites, which are considered hotspots of POLE mutation [10]. Unfortunately, three patients with POLE mutations did not respond to ICIs in the present study. One patient with a P286C mutation was associated with a hypermutation phenotype by NGS, but the other two (with R559W and G1086S mutations) did not show high TMB, although they were tested with different panels (S1 Table), and the identified sites of POLE mutation might not have been hotspots, which could have led to negative results. Further clinical studies with larger sample sizes are necessary to evaluate the activity of ICIs and its association with sites in POLE-mutated CRC.

One of the responders initially showed dMMR by IHC and MSS by PCR at the time of enrollment. However, the IHC result was revised to proficient MMR in the post hoc review. Although NGS could not be performed due to an inadequate 
amount of tumor tissue, leaving the POLE mutation status of this patient unknown, he achieved CR and has continued avelumab treatment for approximately 18 months (Fig. 3). In this regard, there may be unknown factors to explain the mechanism of response to ICIs other than MMR/MSI status or POLE mutation, such as PD-L1 or PD-L2 amplification $[29,30]$, although we could not explore the cause of responsiveness in this patient due to the lack of adequate tumor tissue.

This study has several limitations. The consistency of eligibility and response evaluation could not be fully ensured, because central adjudication of the dMMR/MSI status of tumor tissue and central review of the radiologic response were not performed. Tumor samples were not collected prospectively for research purposes, and therefore translational studies, such as investigation of TMB, tumor-infiltrating lymphocytes, PD-L1 expression, or transcriptome, have not yet been performed. Further post hoc studies are planned with available tissue samples to elucidate their association with the response to ICIs.

In conclusion, avelumab is a promising anti-PD-L1 inhibitor in patients with metastatic or unresectable CRC harboring dMMR/MSI-H or POLE mutations. For the determination of $\mathrm{dMMR} / \mathrm{MSI}-\mathrm{H}$, the conventional IHC method alone appears to be insufficient to select patients who would benefit from immunotherapy. Further studies to identify accurate strategies to select optimal candidates for immu-notherapy are needed.

\section{Electronic Supplementary Material}

Supplementary materials are available at Cancer Research and Treatment website (https://www.e-crt.org).

\section{Conflicts of Interest}

Avelumab was kindly provided by Merck Korea, Seoul, Korea; an affiliate of Merck KGaA, Darmstadt, Germany, as part of an alliance between Merck KGaA and Pfizer. Merck KGaA, Darmstadt, Germany and Pfizer reviewed the manuscript for medical accuracy only before journal submission.

\section{Acknowledgments}

This research was supported by a grant of the Korea Health Technology R\&D Project through the Korea Health Industry Development Institute (KHIDI), funded by the Ministry of Health \& Welfare, Republic of Korea (grant number: HI17C2206).

We thank Dr. Joon Seo Lim from the Scientific Publications Team at Asan Medical Center for his assistance with scientific editing of this manuscript.

\begin{abstract}
Author Details
${ }^{1}$ Department of Oncology, Asan Medical Center, University of Ulsan College of Medicine, Seoul, ${ }^{2}$ Center for Colorectal Cancer, Research Institute and Hospital, National Cancer Center, Goyang, ${ }^{3}$ Division of Medical Oncology, Department of Internal Medicine, Severance Hospital, Yonsei University College of Medicine, Seoul, ${ }^{4}$ Division of Hematology and Medical Oncology, Department of Internal Medicine, Seoul National University Bundang Hospital, Seoul National University College of Medicine, Seongnam, ${ }^{5}$ Department of Internal Medicine, Seoul National University Hospital, Seoul National University College of Medicine, Seoul, ' ${ }^{6}$ Division of Oncology/Hematology, Department of Internal Medicine, Korea University Anam Hospital, Korea University College of Medicine, Seoul, 'Division of Hematology-Oncology, Department of Medicine, Samsung Medical Center, Sungkyunkwan University School of Medicine, Seoul, ${ }^{8}$ Department of Pathology, Asan Medical Center, University of Ulsan College of Medicine, Seoul, Korea
\end{abstract}

\section{References}

1. Jung KW, Won YJ, Kong HJ, Lee ES; Community of Population-Based Regional Cancer Registries. Cancer statistics in Korea: incidence, mortality, survival, and prevalence in 2015. Cancer Res Treat. 2018;50:303-16.

2. Grothey A, Van Cutsem E, Sobrero A, Siena S, Falcone A, Ychou M, et al. Regorafenib monotherapy for previously treated metastatic colorectal cancer (CORRECT): an international, multicentre, randomised, placebo-controlled, phase 3 trial. Lancet. 2013;381:303-12.

3. Mayer RJ, Van Cutsem E, Falcone A, Yoshino T, Garcia-Carbonero R, Mizunuma N, et al. Randomized trial of TAS-102 for refractory metastatic colorectal cancer. N Engl J Med. 2015;372:1909-19.

4. Le DT, Uram JN, Wang H, Bartlett BR, Kemberling H, Eyring $\mathrm{AD}$, et al. PD-1 blockade in tumors with mismatch-repair deficiency. N Engl J Med. 2015;372:2509-20.

5. Overman MJ, McDermott R, Leach JL, Lonardi S, Lenz HJ,
Morse MA, et al. Nivolumab in patients with metastatic DNA mismatch repair-deficient or microsatellite instability-high colorectal cancer (CheckMate 142): an open-label, multicentre, phase 2 study. Lancet Oncol. 2017;18:1182-91.

6. Le DT, Kavan P, Kim TW, Burge ME, Van Cutsem E, Hara H, et al. KEYNOTE-164: Pembrolizumab for patients with advanced microsatellite instability high (MSI-H) colorectal cancer. J Clin Oncol. 2018;36(15 Suppl):3514.

7. Le DT, Kim TW, Van Cutsem E, Geva R, Jager D, Hara H, et al. Phase II open-label study of pembrolizumab in treatmentrefractory, microsatellite instability-high/mismatch repairdeficient metastatic colorectal cancer: KEYNOTE-164. J Clin Oncol. 2020;38:11-9.

8. Llosa NJ, Cruise M, Tam A, Wicks EC, Hechenbleikner EM, Taube JM, et al. The vigorous immune microenvironment of microsatellite instable colon cancer is balanced by multiple counter-inhibitory checkpoints. Cancer Discov. 2015;5:43-51. 
9. Koopman M, Kortman GA, Mekenkamp L, Ligtenberg MJ, Hoogerbrugge N, Antonini NF, et al. Deficient mismatch repair system in patients with sporadic advanced colorectal cancer. Br J Cancer. 2009;100:266-73.

10. Palles C, Cazier JB, Howarth KM, Domingo E, Jones AM, Broderick $\mathrm{P}$, et al. Germline mutations affecting the proofreading domains of POLE and POLD1 predispose to colorectal adenomas and carcinomas. Nat Genet. 2013;45:136-44.

11. Kaufman HL, Russell J, Hamid O, Bhatia S, Terheyden P, D'Angelo SP, et al. Avelumab in patients with chemotherapyrefractory metastatic Merkel cell carcinoma: a multicentre, single-group, open-label, phase 2 trial. Lancet Oncol. 2016;17: 1374-85.

12. Vaishampayan U, Schoffski P, Ravaud A, Borel C, Peguero J, Chaves J, et al. Avelumab monotherapy as first-line or secondline treatment in patients with metastatic renal cell carcinoma: phase Ib results from the JAVELIN Solid Tumor trial. J Immu-nother Cancer. 2019;7:275.

13. Patel MR, Ellerton J, Infante JR, Agrawal M, Gordon M, Aljumaily R, et al. Avelumab in metastatic urothelial carcinoma after platinum failure (JAVELIN Solid Tumor): pooled results from two expansion cohorts of an open-label, phase 1 trial. Lancet Oncol. 2018;19:51-64.

14. Disis ML, Taylor MH, Kelly K, Beck JT, Gordon M, Moore KM, et al. Efficacy and safety of avelumab for patients with recurrent or refractory ovarian cancer: phase $1 \mathrm{~b}$ results from the JAVELIN solid tumor trial. JAMA Oncol. 2019;5:393-401.

15. Gulley JL, Rajan A, Spigel DR, Iannotti N, Chandler J, Wong DJ, et al. Avelumab for patients with previously treated metastatic or recurrent non-small-cell lung cancer (JAVELIN Solid Tumor): dose-expansion cohort of a multicentre, open-label, phase $1 b$ trial. Lancet Oncol. 2017;18:599-610.

16. Choi YJ, Kim YH. The cancer precision medicine diagnosis and treatment (K-MASTER) enterprise. Korean J Med. 2019;94:246-51.

17. Kelly K, Infante JR, Taylor MH, Patel MR, Wong DJ, Iannotti $\mathrm{N}$, et al. Safety profile of avelumab in patients with advanced solid tumors: a pooled analysis of data from the phase 1 JAVELIN solid tumor and phase 2 JAVELIN Merkel 200 clinical trials. Cancer. 2018;124:2010-7.

18. Heery CR, O'Sullivan-Coyne G, Madan RA, Cordes L, Rajan A, Rauckhorst M, et al. Avelumab for metastatic or locally advanced previously treated solid tumours (JAVELIN Solid Tumor): a phase 1a, multicohort, dose-escalation trial. Lancet Oncol. 2017;18:587-98.

19. Fuchs CS, Doi T, Jang RW, Muro K, Satoh T, Machado M, et al. Safety and efficacy of pembrolizumab monotherapy in patients with previously treated advanced gastric and gastroe- sophageal junction cancer: phase 2 clinical KEYNOTE-059 trial. JAMA Oncol. 2018;4:e180013.

20. Marabelle A, Le DT, Ascierto PA, Di Giacomo AM, De JesusAcosta A, Delord JP, et al. Efficacy of pembrolizumab in patients with noncolorectal high microsatellite instability/mismatch repair-deficient cancer: results from the phase II KEYNOTE-158 study. J Clin Oncol. 2020;38:1-10.

21. Kelderman S, Schumacher TN, Kvistborg P. Mismatch repairdeficient cancers are targets for anti-PD-1 therapy. Cancer Cell. 2015;28:11-3.

22. Schrock AB, Ouyang C, Sandhu J, Sokol E, Jin D, Ross JS, et al. Tumor mutational burden is predictive of response to immune checkpoint inhibitors in MSI-high metastatic colorectal cancer. Ann Oncol. 2019;30:1096-103.

23. Mandal R, Samstein RM, Lee KW, Havel JJ, Wang H, Krishna $\mathrm{C}$, et al. Genetic diversity of tumors with mismatch repair deficiency influences anti-PD-1 immunotherapy response. Science. 2019;364:485-91.

24. Grasso CS, Giannakis M, Wells DK, Hamada T, Mu XJ, Quist $\mathrm{M}$, et al. Genetic mechanisms of immune evasion in colorectal cancer. Cancer Discov. 2018;8:730-49.

25. Cohen R, Hain E, Buhard O, Guilloux A, Bardier A, Kaci R, et al. Association of primary resistance to immune checkpoint inhibitors in metastatic colorectal cancer with misdiagnosis of microsatellite instability or mismatch repair deficiency status. JAMA Oncol. 2019;5:551-5.

26. Klarskov L, Ladelund S, Holck S, Roenlund K, Lindebjerg J, Elebro J, et al. Interobserver variability in the evaluation of mismatch repair protein immunostaining. Hum Pathol. 2010; 41:1387-96.

27. Konstantinopoulos PA, Luo W, Liu JF, Gulhan DC, Krasner C, Ishizuka JJ, et al. Phase II study of avelumab in patients with mismatch repair deficient and mismatch repair proficient recurrent/persistent endometrial cancer. J Clin Oncol. 2019;37: 2786-94.

28. Gong J, Wang C, Lee PP, Chu P, Fakih M. Response to PD-1 blockade in microsatellite stable metastatic colorectal cancer harboring a POLE mutation. J Natl Compr Canc Netw. 2017; 15:142-7.

29. Sorscher S, Resnick J, Goodman M. First case report of a dramatic radiographic response to a checkpoint inhibitor in a patient with proficient mismatch repair gene expressing metastatic colorectal cancer. JCO Precis Oncol. 2017 Feb 23 [Epub]. https://doi.org/10.1200/PO.16.00005.

30. Goodman AM, Piccioni D, Kato S, Boichard A, Wang HY, Frampton G, et al. Prevalence of PDL1 amplification and preliminary response to immune checkpoint blockade in solid tumors. JAMA Oncol. 2018;4:1237-44. 